\title{
Uma Análise do Comportamento de Algumas Medidas de Ajuste Usadas na Determinação dos Parâmetros de Modelos de Distribuição de Viagens
}

E.T. BEZ, Centro de Educação Superior VII, Universidade do Vale do Itajaí, UNIVALI, 88122-000 São José, SC, Brasil

M.B. GONÇALVES, Departamento de Matemática, Centro de Ciências Físicas e Matemáticas, UFSC, 88010-970 Florianópolis, SC, Brasil.

\begin{abstract}
Resumo. Modelos de distribuição de viagens são usados no contexto de Planejamento de Transportes para estimar o número de viagens entre pares de zonas de tráfego numa área de estudo. Em geral, estes modelos possuem parâmetros que devem ser determinados para cada situação particular. Para determinar esses parâmetros algumas medidas clássicas de ajuste são usadas, tais como: princípio estatístico da máxima verossimilhança, soma média dos erros quadrados e estatística $\phi$-normalizada.

$\mathrm{Na}$ literatura encontram-se relatos de que, dependendo da medida de ajuste usada, a determinação dos parâmetros de alguns modelos pode constituir-se em verdadeiro pesadelo computacional. Neste contexto, no presente trabalho procura-se investigar o comportamento dessas medidas bem como as principais dificuldades associadas aos procedimentos numéricos utilizados para a determinação dos parâmetros de modelos gravitacionais e gravitacionais de oportunidades, os quais constituem a classe mais difundida de modelos de distribuição de viagens.
\end{abstract}

\section{Introdução}

Uma parte importante do processo de Planejamento de Transportes é a estimativa do número provável de viagens, no presente e em alguma data futura, entre as diversas partes (zonas de tráfego) que compõem uma área de estudo.

Nesta fase do Planejamento de Transportes, chamada fase de distribuição de viagens, os modelos matemáticos exercem um papel preponderante. Destacamse os modelos gravitacionais, que foram concebidos de forma empírica através de analogia com a lei da gravidade de Newton, tendo recebido, posteriormente, um embasamento teórico fundamentado no principio da maximização da entropia [18].

Paralelamente ao desenvolvimento dos modelos gravitacionais, diversos pesquisadores se empenharam na construção de modelos alternativos, entre os quais destaca-se o modelo de oportunidades intervenientes de Schneider [6]. 
Posteriormente, modelos híbridos [16] e [8], denominados modelos gravitacionais de oportunidades, foram concebidos com o objetivo de produzir estimativas mais precisas dos padrões de interação espacial. No entanto, muitos aspectos a eles relacionados não foram ainda suficientemente estudados. Um desses aspectos diz respeito à calibração, que é de extrema importância no processo de modelagem.

O critério de calibração mais freqüente para a determinação dos parâmetros dos modelos gravitacionais e/ou híbridos baseia-se no princípio estatístico da máxima verossimilhança [5], [7] e [19]. No entanto, outras estatísticas também são utilizadas, tais como a soma média dos erros quadrados [4] ou a estatística $\phi$-normalizada [15].

Estudos realizados por [4], [9] e [11] entre outros, demonstram que, freqüentemente, o processo de calibração dos modelos está associado a dificuldades numéricas como o surgimento de over e/ou underflow e a não convexidade da função a ser minimizada.

Neste contexto o presente estudo foi elaborado com o intuito de investigar o comportamento das medidas de ajuste citadas e a influência das dificuldades numéricas sobre a robustez dos métodos utilizados na determinação dos parâmetros de modelos gravitacionais e gravitacionais de oportunidades.

\section{Modelos de Distribuição de Viagens}

No presente trabalho são enfocados os modelos gravitacional entrópico [17] e gravitacional de oportunidades [7]. São consideradas versões duplamente restritas, que contemplam as restrições de consistência de fluxo na origem e no destino e simplesmente restritas, com restrição de origem, considerando ou não a atratividade.

A seguinte notação é utilizada: $i$ representa uma zona de origem; $j$ representa uma zona de destino; $O_{i}$ é uma medida do número de viagens que se originam na zona $i ; D_{j}$ é uma medida do número de viagens atraídas pela zona $j ; T_{i j}{ }^{*}$ é o número de viagens observado por célula; $T^{*}$ é o número total de viagens observadas; $T$ é o número total de viagens estimadas; ncel é o número de células da matriz com fluxo estimado; $T_{i j}$ é o número de viagens que se originam em $i$ e terminam em $j$; $\mathrm{w}_{i j}$ é uma medida do número de oportunidades que se interpõe entre as zonas $i$ e $j$; $\mathrm{c}_{i j}$ é uma medida de separação espacial entre as zonas $i$ e $j ; n$ representa o número de zonas de tráfego da área de estudo.

As versões duplamente restrita e simplesmente restritas analisadas do modelo gravitacional entrópico, são descritas em [7] e [10].

Os casos do Modelo Gravitacional - de Oportunidades, Duplamente Restrito (MGODR), Simplesmente Restrito com restrição de Origem (MGOSRO) e Simplesmente Restrito com restrição de Origem, considerando a atratividade (MGOSROA) representados pelas equações $(2.1)$, (2.2) e (2.3) também foram objeto do nosso estudo,

$$
\begin{gathered}
T_{i j}=A_{i} B_{j} O_{i} D_{j} e^{-\left(\beta \cdot c_{i j}+\lambda w_{i j}\right)} ; \\
T_{i j}=A_{i} O_{i} e^{-\left(\beta \cdot c_{i j}+\lambda w_{i j}\right)} ;
\end{gathered}
$$




$$
T_{i j}=A_{i} O_{i} D_{j} e^{-\left(\beta \cdot c_{i j}+\lambda w_{i j}\right)}
$$

onde $\mathrm{A}_{i}, \mathrm{~B}_{j}, \beta$ e $\lambda$ são parâmetros a serem determinados.

\section{Medidas de Ajuste e Procedimentos Numéricos}

Neste estudo, foram utilizados como critérios de calibração o princípio estatístico da máxima verossimilhança [5] e [8], a soma média dos erros quadrados [4] e a estatística $\phi$-Normalizada [15], para estimar os parâmetros do modelo gravitacional e do modelo gravitacional - de oportunidades.

Utilizando o princípio da máxima verossimilhança, [5] obteve as seguintes equações para a determinação dos parâmetros do modelo gravitacional duplamente restrito.

$$
\begin{aligned}
& A_{i}=\left[\sum_{j} B_{j} D_{j} e^{-\beta \cdot c_{i j}}\right]^{-1} ; \\
& B_{j}=\left[\sum_{i} A_{i} O_{i} e^{-\beta \cdot c_{i j}}\right]^{-1} ; \\
& \sum_{i j} \frac{T_{i j}^{*}}{T^{*}} c_{i j}=\sum_{i j} \frac{T_{i j}}{T} c_{i j} .
\end{aligned}
$$

As equações (3.1) e (3.2) constituem um sistema não linear de $2 n$ equações cujas incógnitas $\mathrm{A}_{i}$ e $\mathrm{B}_{j}$ são chamadas fatores de balanceamento e são determinadas de forma a garantir as equações de consistência de fluxo $\sum_{j} T_{i j}=O_{i}$ e $\sum_{i} T_{i j}=D_{j}$.

A equação (3.3) é usada para determinar o parâmetro $\beta$, chamado parâmetro de impedância.

O princípio estatístico da máxima verossimilhança também foi usado por [7] para a determinação dos parâmetros do modelo gravitacional - de oportunidades duplamente restrito (equação (2.1)), que obteve resultados análogos aos obtidos por [5] para o modelo gravitacional. As seguintes equações foram obtidas por [8]:

$$
\begin{gathered}
A_{i}=\left[\sum_{j} B_{j} D_{j} e^{-\left(\beta \cdot c_{i j}+\lambda w_{i j}\right)}\right]^{-1} ; \\
B_{j}=\left[\sum_{i} A_{i} O_{i} e^{-\left(\beta \cdot c_{i j}+\lambda w_{i j}\right)}\right]^{-1} ; \\
\sum_{i j} \frac{T_{i j}^{*}}{T^{*}} c_{i j}=\sum_{i j} \frac{T_{i j}}{T} c_{i j} ; \\
\sum_{i j} \frac{T_{i j}^{*}}{T^{*}} w_{i j}=\sum_{i j} \frac{T_{i j}}{T} w_{i j} .
\end{gathered}
$$

Em nosso estudo os fatores de balanceamento $\mathrm{A}_{i}$ e $\mathrm{B}_{j}$ foram determinados através do método de equilíbrio de matrizes de Furness. 
O parâmetro de impedância $\beta$, para o caso das versões utilizadas do modelo gravitacional entrópico, foi obtido de forma a reproduzir o custo médio de viagens observado (equação (3.3)), através do método das secantes [12].

Os parâmetros de impedância $\beta$ e $\lambda$, para os modelos gravitacionais de oportunidades descritos na seção anterior, foram determinados de forma a reproduzir o custo médio de viagens observado e o número médio observado de oportunidades intervenientes por viagem (equações (3.6) e (3.7)), através da minimização da função

$$
F(\beta, \lambda)=\left(\bar{c}-\sum_{i j} \frac{T_{i j}(\beta, \lambda)}{T} \cdot c_{i j}\right)^{2}+\left(\bar{w}-\sum_{i j} \frac{T_{i j}(\beta, \lambda)}{T} \cdot w_{i j}\right)^{2}
$$

onde

$$
\bar{c}=\frac{\sum_{i j} T_{i j}^{*} c_{i j}}{T^{*}} e^{\bar{w}}=\frac{\sum_{i j} T_{i j}^{*} w_{i j}}{T^{*}} .
$$

A estatística soma média dos erros quadrados, ou seja, "Mean Sum of Squares Error" foi utilizada por [4] num estudo sobre o uso de algoritmos genéticos para calibração de modelos de distribuição de viagens. A equação que representa esta estatística é dada pela seguinte expressão:

$$
f(\text { error })=\sum_{i j} \frac{\left(T_{i j}^{*}-T_{i j}\right)^{2}}{n c e l}
$$

A terceira medida de ajuste utilizada, como critério de calibração, foi a estatística $\phi$-Normalizada apresentada por [15] na calibração de modelos de distribuição de viagens. A expressão que representa esta estatística, é dada por:

$$
f=\sum_{i j} \frac{T_{i j}^{*}}{T^{*}}\left|\ln \left(\frac{T_{i j}^{*}}{T_{i j}}\right)\right|
$$

Tanto para a soma média dos erros quadrados quanto para a estatística $\phi$-Normalizada foi utilizada, para determinação do parâmetro $\beta$, a Busca de Fibonacci [13], nos três casos do modelo gravitacional.

Para a determinação dos parâmetros $\beta$ e $\lambda$ do modelo gravitacional - de oportunidades, as funções (3.8), (3.10) e (3.11) foram minimizadas através do Método do Gradiente com Perturbações Aleatórias [14], [9] e [11], o qual busca evitar a convergência para mínimos locais adicionando perturbações aleatórias adequadas à relação de recorrência do método do gradiente, com o intuito de garantir a convergência independentemente do ponto inicial que for tomado [14]. Ou seja, $x_{n+1}=$ $Q\left(x_{n}\right)+P_{n}$ onde, $P_{n}$ é uma perturbação aleatória que decresce de forma lenta de modo a evitar a convergência para um mínimo local. A implementação deste método pode ser feita seguindo os passos do seguinte algoritmo [9] e [11]: Inicialmente geramos uma seqüência $\left(x_{n}\right)_{n>1}$, de modo que, $x_{n+1}=Q\left(x_{n}\right)+P_{n}, \forall n \geq 0$ onde, $Q(x)=x-\mu \cdot \nabla F(x)$. Em seguida, escolhe-se um número inteiro positivo NR, e no passo n, $x_{n+1}$ é obtido através de $x_{n+1}=\operatorname{Arg} \min \left\{F\left(Q\left(x_{n}\right)+P_{n}^{r}\right) ; 0 \leq r \leq N R\right\}$ 
onde, $P_{n}^{0}=0$ e $P_{n}^{1}, P_{n}^{2}, ., P_{n}^{N R}$ são $\mathrm{NR}$ valores aleatórios de $P_{n}$. Para cada r, $1 \leq r \leq N R$, o vetor $P_{n}^{r}$ é dado por $P_{n}^{r}=\lambda_{n} Z_{n}^{r}$, sendo $\left(\lambda_{n}\right)_{n \geq 1}$ uma seqüência de números reais convergindo para zero, gerada pela expressão: $\lambda_{n}=a \sqrt{\frac{c}{\log (n+d)}}$ em que, a, c e d são constantes positivas, e $\left(Z_{n}^{r}\right)$ é um vetor gerado através da distribuição gaussiana padronizada $\mathrm{N}$-dimensional $N(0, I d)$, onde Id é uma matriz identidade.

\section{Conjunto de Dados}

Para realização dos testes numéricos, fizemos uso de um conjunto de dados coletado pela Pesquisadora Lourdes Maria Werle de Almeida [1], na cidade de Londrina Paraná e referem-se a viagens de estudantes de ensino médio às escolas.

Uma matriz observada $\left[T_{i j}^{*}\right]_{12 \times 12}$ de distribuição de viagens de estudantes às escolas foi obtida por [1], através de um formulário distribuído em 29 escolas, distribuídas em 12 zonas. Estas zonas foram determinadas, com base em dados fornecidos pelo Instituto de Planejamento Urbano de Londrina (IPPUL).

Uma matriz de separação espacial $\left[c_{i j}\right]_{12 x 12}$ foi determinada através do tempo que o estudante levava para se deslocar de sua residência até a escola, incluindo o tempo de espera e de caminhada, utilizando ônibus como meio de transporte.

Uma matriz de oportunidades intervenientes $\left[w_{i j}\right]_{12 x 12}$ foi obtida através de metodologia desenvolvida por Almeida [1] e [2], onde são identificados os principais atributos considerados na escolha das escolas pelos estudantes.

Este conjunto de dados foi utilizado num estudo anterior [3], onde o objetivo era testar os critérios de calibração descritos na seção 3 e verificar a sua influência na precisão das estimativas apresentadas pelos modelos de distribuição de viagens, descritos na seção 2. Embora o objetivo desse estudo não fosse o de analisar o comportamento das medidas de ajuste usadas como critério de calibração, foram observadas algumas características interessantes, já comentadas na literatura, como a não convexidade das funções a serem otimizadas, localização do ótimo global em fenda profunda e estreita, entre outras.

Surgiu, então, a seguinte questão: As características observadas seriam peculiares ao conjunto de dados ou inerentes às medidas de ajuste utilizadas? Com o objetivo de investigar esta questão e a robustez dos procedimentos numéricos usados, resolvemos simular novas matrizes de viagens.

Para isto, fizemos uso do software MATLAB para Windows versão 5.1.0.421, usando a função rand(12,12). Esta função gera uma matriz quadrada de números aleatórios, de ordem 12, uniformemente distribuídos entre 0 e 1 . Para tornar as entradas compatíveis com possíveis situações reais multiplicamos a matriz por 1000 (mil) e fizemos uso da função "round" para arredondar as entradas da matriz. O simulador de números aleatórios do MATLAB mantém a consistência de fluxo da matriz, tanto na origem quanto no destino. Assim, cada matriz simulada representa uma possível matriz de viagens observada para a área de estudo. As matrizes de separação espacial e de oportunidades intervenientes coletadas por [1] foram associadas a estas matrizes simuladas. 


\section{Análise do Comportamento das Funções a serem Minimizadas}

Fazendo uso destes dados simulados em conjunto com os dados reais coletados por [1], plotamos os gráficos das funções (3.8), (3.10) e (3.11) no MATLAB, versão especificada na seção anterior.

Para o princípio de máxima verossimilhança (função (3.8)), a não convexidade das funções juntamente com a localização do ponto de ótimo global da função em fendas estreitas, foi uma constante nos dados simulados. As características percebidas com dados reais (ver Figuras 1 e 2) foram, também, detectadas com os dados simulados.

Os gráficos plotados para a estatística $\phi$-normalizada demonstram que a função não tem problema com relação a sua convexidade. Entretanto, percebemos que em algumas regiões a função tem como característica marcante sua superfície extremamente plana. Isso não é uma boa característica para uma medida usada como critério de calibração pois no processo de otimização, para a mesma situação, a parada pode acontecer para valores bem distintos de beta e lambda.

Para a estatística da soma média dos erros quadrados, as características verificadas em [3], também foram detectadas, quando fizemos uso dos dados simulados. O grande problema detectado diz respeito a não convexidade (ver Figuras 3 e 4).

\section{Análise da Robustez dos Procedimentos de Calibração Utilizados}

Verificado o comportamento das funções a serem minimizadas, executamos os testes numéricos de calibração. Os métodos numéricos citados na seção 3 foram implementados no MATLAB, versão especificada na seção 4. Os problemas surgiram, com freqüência, quando passamos a calibrar modelos híbridos. Superfícies com "bom comportamento", isto é, convexas como a estatística $\phi$-normalizada, não necessariamente determinaram um bom ajuste, devido a forma plana apresentada. Um outro problema verificado é a forma exponencial apresentada nas funções a serem otimizadas.

Os testes foram realizados com variações no ponto inicial, no número gerado de perturbações a cada passo do gradiente e na quantidade de passos do gradiente, antes de gerar uma nova perturbação. Os valores destas variações são os seguintes: Pontos iniciais: $(0,0)(1,0)(0,1)(1,1)$; Número de perturbações (NP): 0, 5, 10, 30; Passos antes de perturbar(PAP) : $1,5,10$; Valores das constantes: $\mu=0.1 ; \mathrm{a}=0.1$, $\mathrm{d}=1, \mathrm{c}=1$. Um fator de redução de 0.9 para $\mu$ e de 0.7 para $a$, foi aplicado a cada 10 iterações. O Critério de parada: número de iterações NIT $=800$ ou $\epsilon<10^{-3}$ ou se os últimos 40 valores da função forem iguais. Em virtude dos maiores problemas serem ocasionados durante a calibração dos modelos híbridos, nos concentramos em testá-los, combinando os "pontos", "número de permutações" e "passos antes de perturbar", com o intuito de observarmos as dificuldades que poderiam ser geradas. 


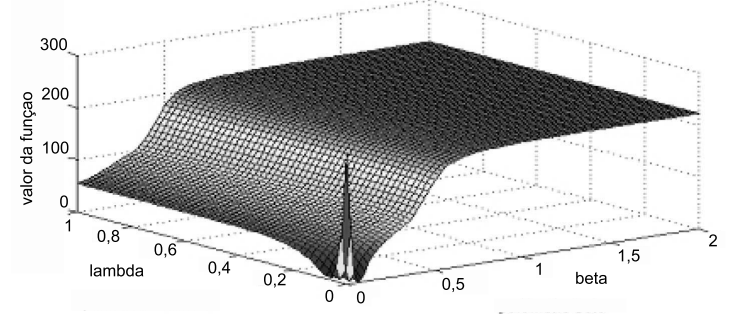

Figura 1: MGOSROA - Critério MV; retângulo [0;2]x[0;1]; int. da malha de 0.02 .

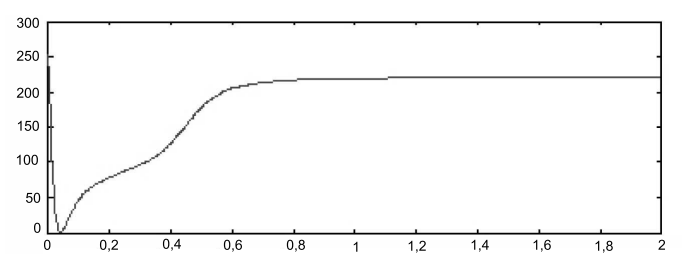

Figura 2: Corte da superfície de $F(\beta, \lambda) ; \beta=2 \lambda$ intervalo [0,2] passo 0.001; MGOSROA - Critério MV

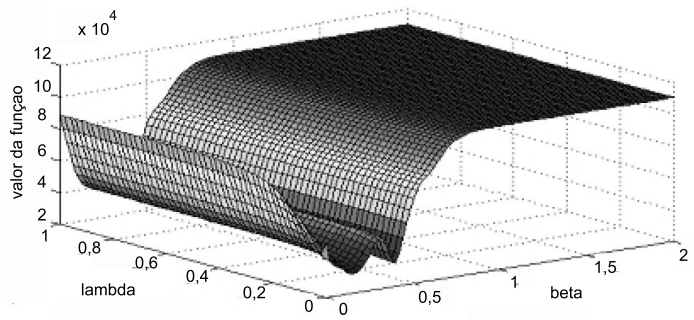

Figura 3: MGOSROA - Critério SMEQ; retângulo [0;2]x[0;1]; int. da malha de 0.02.

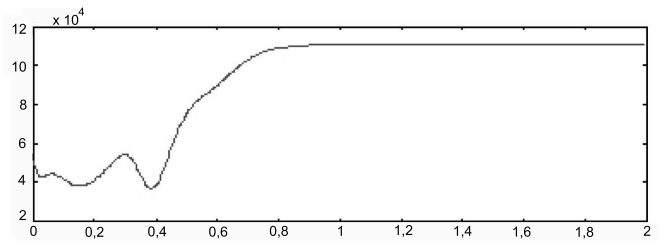

Figura 4: Corte da superfície de $F(\beta, \lambda) ; \beta=2 \lambda$ no int. [0, 2] passo 0.001;MGOSROA - Critério SMEQ 
Foram realizados em torno de 500 (quinhentos) testes numéricos com o objetivo de pesquisar a influência que as variações dos parâmetros de inicialização exercem no ajuste das matrizes.

A variação do NP, foi uma tentativa de gerar um número maior de vetores aleatórios, o que possibilitaria, teoricamente, um número maior de opções a serem testadas, criando uma melhor expectativa na busca do ótimo global.

O aumento do NP não demonstrou eficiência na busca do ótimo global. Da mesma forma, que a perturbação é utilizada para facilitar na busca do ótimo global, o aumento de NP torna o processo mais lento, devido ao aumento do número de avaliações da função objetivo. É importante notar que, para a versão duplamente restrita, cada avaliação da função objetivo desencadeia a método de equilíbrio de matrizes para a determinação dos $\mathrm{A}_{i}$ e dos $\mathrm{B}_{j}$. O valor que se mostrou mais adequado entre os testados foi $\mathrm{NP}=5$.

A idéia de testar PAP foi uma tentativa de mesclar o método do gradiente ("caminhar" na direção de decrescimento da função sem gerar perturbações aleatórias), com o método do gradiente com perturbações aleatórias, visando diminuir o número de iterações do processo.

A utilização do PAP em muitos momentos criava um problema maior que o já existente, haja visto que em muitos casos a utilização do PAP fazia com que buscássemos mais rapidamente o mínimo local, impossibilitando uma fuga a tempo, na busca do mínimo global.

Outro fato a ser descrito é que, quando consideramos a minimização irrestrita, percebemos que, em muitos casos, a posição do ótimo da função era determinada por valores negativos de beta e lambda. Entretanto apenas valores positivos destes parâmetros apresentam consistência teórica para o problema estudado.

Os testes realizados variando o ponto inicial demonstraram que, para algumas escolhas do mesmo, não foi alcançada a convergência para o mínimo global. Isso ocorreu especialmente para o modelo (2.3), e as funções (3.8) e (3.10), para o ponto $(1,1)$.

\section{Conclusões}

As funções a serem minimizadas apresentaram as mesmas características para o conjunto de dados reais e para os diversos conjuntos de dados simulados. Isso é um indicativo de que estas características são inerentes às medidas de ajuste usadas e não ao conjunto de dados.

As dificuldades numéricas ocorridas durante a calibração puderam ser superadas com a percepção do melhor ponto de inicialização do processo, no caso o ponto $(0,0)$ tendo sido alcançada a convergência global em todos os casos testados.

Uma análise geral dos resultados obtidos aponta como robustos os procedimentos de calibração associados ao princípio estatístico da máxima verossimilhança.

Finalmente, observamos que uma das maiores dificuldades na obtenção dos parâmetros beta e lambda é a inicialização adequada dos parâmetros dos algoritmos utilizados. Isso é um fator negativo, pois um pacote para a calibração desses modelos deveria ser de fácil utilização por profissionais da área de Planejamento 
de Transportes, os quais, em geral, não dominam as técnicas de otimização. Dessa forma, seria interessante testar outros métodos de otimização global para a resolução dos problemas apresentados.

Abstract. Trip distribution models are used in the Transport Planning context to estimate the number of trips between traffic zones in a study area. In general these models have parameters that have to be determined to each particular situation. In order to determine these parameters some classical adjustment measurements are used, such as statistic principle of maximum likelihood, average sum of square error and $\phi$-normalized statistic.

Some accounts are found in literature in which, depending on the adjustment measurement used, the parameters determination of some models may become a real computational nightmare. This essay tries to investigate, within this context, the behaviour of these measurements, as well as the main difficulties associated to the numerical procedures used to determine the parameters of the gravitational and opportunity gravitational models. These two are the most known trip distribution models.

\section{Referências}

[1] L.M.W. Almeida, "Desenvolvimento de uma Metodologia para Análise Locacional de Sistemas Educacionais Usando Modelos de Interação Espacial e Indicadores de Acessibilidade", Tese de Doutorado, PPGEP, UFSC, Florianópolis, SC, 1999.

[2] L.M.W. Almeida e M.B. Gonçalves, A Methodology to incorporate behavioral aspects in trip-distribution models with an application to estimate student flow, Environment and Planning A, 33 (2001), 1125-1138.

[3] E.T. Bez e M.B. Gonçalves, Um estudo sobre os procedimentos usados para a determinação dos parâmetros de alguns modelos de distribuição de viagens, em "Memorias do XI CLATPU", Habana, Cetra, 2001. 1 CD.

[4] G. Diplock e S. Openshaw, Using simple genetic algorithms to calibrate spatial interaction models, Geographical Analysis, 28, No. 3 (1996), 262-279.

[5] A.W. Evans, The calibration of trip distribution models with exponential or similar cost functions, Transportation Research, 5 (1971), 15-38.

[6] N.D. Finney, "Trip Distribution Models: New Perspectives in Urban Transportation Research", A.J. Catanese, Lexington: Lexington Books, pp. 63-146, 1972 .

[7] M.B. Gonçalves, "Desenvolvimento e Teste de um Novo Modelo Gravitacional de Oportunidades de Distribuição de Viagens", Tese de Doutorado, PPGEP, UFSC, Florianópolis, SC, 1992. 
[8] M.B. Gonçalves e I. Ulysséa Neto, The development of a new gravityopportunity model for trip distribution, Environment and Planning A, 25 (1993), 817-826.

[9] M.B. Gonçalves e J.E. Souza de Cursi, Métodos robustos para a calibração de modelos de interação espacial em transportes, em "Anais do XI ANPET", Vol. 2, pp. 303-313, 1997.

[10] M.B. Gonçalves, L.M.W. Almeida e E.T. Bez, Análise comparativa de alguns modelos de distribuição de viagens usados para estimar fluxos de passageiros por motivo escola, em "Anais do XI PANAM", pp 467-480, 2000.

[11] M.B. Gonçalves e J.E. Souza de Cursi, Parameter estimation in a trip distribution model by random perturbation of a descent method, Transportation Research B, 35 (2001), 137-161.

[12] G.M. Hyman, The calibration of trip distribution models, Environment and Planing, 1 (1969), 105-112.

[13] A.G. Novaes "Métodos de Otimização: Aplicações aos Transportes", Edgard Blücher, São Paulo, 1978.

[14] M. Pogu e J.E. Souza de Cursi, Global optimization by random perturbation of the gradient method with a fixed parameter, Journal of Global Optimization, 5 (1994), 159-180.

[15] D.P. Smith e R.G. Hutchinson, Goodness of fit statistics for trip distribuition models, Transportation Research, 15A (1981), 295-303.

[16] M.J. Wills, A flexible gravity-opportunities model for trip distribution, Transportation Research, 20B (1986), 89-111.

[17] A.G. Wilson, A statistical theory of spatial distribution models, Transportation Research, 1 (1967), 253-269.

[18] A.G. Wilson, "Entropy in Urban and Regional Modelling", Pion, London, 1970.

[19] S. Yun e A. Sen, Computation of maximum likelihood estimates of gravity model parameters, Journal of Regional Science, 34, No. 2 (1994), 199-216. 